\title{
STUDY OF A DIFFERENTIAL OPERATOR OF HEUN TYPE ARISING IN FLUID DYNAMICS
}

\author{
Marina ChugunOVA AND HANS VOLKMER
}

Abstract. The paper studies the non-selfadjoint linear differential operator

$$
L y=\frac{d}{d t}\left((1-a \cos t) y+b \sin t \frac{d y}{d t}\right)
$$

acting in the Hilbert space $L^{2}(-\pi, \pi)$ that originated from a steady state stability problem in fluid dynamics. The operator $L$ is of Heun type and involves two parameters $a, b$ related to the hydrostatic pressure and capillary properties of the fluid. The results concern (1) the properties of functions in the domain of definition of $L$, (2) conditions on $a, b$ for the linear span of the Fourier basis $\left\{e^{i n t}\right\}$ to be core of $L$, and (3) the matrix representation of the reduced resolvent of $L$ in the Fourier basis. In particular, it is shown that the reduced resolvent is compact and of trace class $\mathscr{S}_{1}$.

Mathematics subject classification (2000): 33E10, 47B10, 34L10.

Keywords and phrases: internal singularity, Heun equation, reduced resolvent, core of an operator, Schatten class.

\section{REFERENCES}

[1] E. S. Benilov, S. B. G. O'BRIEn AND I. A. Sazonov, A new type of instability: explosive disturbances in a liquid fild inside a rotating horizontal cylinder, J. Fluid Mech. 497, (2003), 201-224.

[2] E. S. Benilov, M. S. Benilov And N. Kopteva, Steady rimming flows with surface tension, J. Fluid Mech. 597 (2008), 91-118.

[3] M. Chugunova, I. M. Karabash and S. G. Pyatkov, On the nature of ill-posedness of the forward-backward heat equation, preprint, arXiv:0803.2552v2 [math.AP] (2008).

[4] M. Chugunova, D. Pelinovs Ky, Spectrum of a non-self-adjoint operator associated with the periodic heat equation, J. Math. Anal. Appl., 342 (2008), 970-988.

[5] M. Chugunova, V. Strauss, Factorization of an indefinite convection-diffusion operator, C. R. Math. Rep. Acad. Sci. Canada, 30, 2 (2008), 40-47.

[6] M. Chugunova, V. Strauss, On Factorization of a Perturbation of a J-selfadjoint Operator Arising in Fluid Dynamics, arXiv:0809.1555, (2008).

[7] M. Chugunova And H. Volkmer, Spectral analysis of an operator arising in fluid dynamics, to appear in Studies of Applied Math (2009).

[8] E. B. DAVIS, An indefinite convection-diffusion operator, LMS J. Comput. Math. 10 (2007), 288-306.

[9] I. Gohberg AND M. G. KREIN, Introduction to the Theory of Linear Non-selfadjoint Operators, AMS Translations, Providence, 18 (1969).

[10] I. Gohberg, S. Goldberg And N. Krupnik, Traces and Determinants of Linear Operators, Birkhäuser Verlag, Basel Boston Berlin, 2000.

[11] I. GohBerg, A. MARKus, On some relations between eigenvalues and matrix elements of linear operators, Math. sb. 64 (106) (1964), 481-496, English transl. in Amer. Soc. Transl. (2), 52 (1966).

[12] T. Kato, Perturbation Theory for Linear Operators, Springer-Verlag, Berlin Heidelberg New York, 1980.

[13] H. K. MofFatT, Behaviour of a viscous film on the outer surface of a rotating cylinder, J. Mec. 187 (1977), 651-673. 
[14] F. W. Olver, Asymptotics and Special Functions, A K Peters, Natick, Massachusetts, 1997.

[15] A. Zygmund, Trigonometric Series, Cambridge University Press, 1959. 